\title{
Dysregulation of miRNAs and their potential as biomarkers for the diagnosis of gastric cancer
}

\author{
BO GUO ${ }^{1}$, JIE LI $^{2}$, LIYING LIU ${ }^{1}$, NI HOU ${ }^{1}$, DONGMIN CHANG ${ }^{4}$, LINGYU ZHAO ${ }^{1}$, \\ ZONGFANG $\mathrm{LI}^{5}$, TUSHENG SONG ${ }^{1}$ and CHEN HUANG ${ }^{1,3}$
}

\author{
${ }^{1}$ Department of Genetics and Molecular Biology, Key Laboratory of Environment and Genes Related to Diseases, \\ Ministry of Education, Medical College, Xi'an Jiaotong University, Xi'an; ${ }^{2}$ The Second Affiliated Hospital of Shaanxi \\ University of Traditional Chinese Medicine, Xianyang; ${ }^{3}$ Cardiovascular Research Center, Medical College, \\ Xi'an Jiaotong University; ${ }^{4}$ Department of Tumor Surgery, The First Affiliated Hospital of Medical College, \\ Xi'an Jiaotong University; ${ }^{5}$ Engineering Research Center of Biotherapy and \\ Translational Medicine of Shaanxi, Xi'an, Shaanxi, P.R. China
}

Received August 09, 2013; Accepted September 18, 2013

DOI: $10.3892 /$ br.2013.175

\begin{abstract}
Recent studies demonstrated that microRNA (miRNA) expression is dysregulated in numerous human cancers. In this study, we investigated the expression patterns of 8 miRNAs in gastric cancer and evaluated their clinical significance in order to identify potential biomarkers for gastric cancer diagnosis. Total RNA was extracted from gastric cancer and normal tissues from 20 pairs of paraffin-embedded specimens. The expression levels of the miRNAs were detected by quantitative reverse transcriptase polymerase chain reaction using specific stem-loop primers, with U6 as the internal reference gene. The association between miRNA expression level and clinicopathological factors was investigated. The expression of miR-21, -103, -106a, -221 and -222 in gastric cancer samples was significantly higher compared to that in the paired normal samples. Conversely, the expression of miR-143 and -195 in cancer tissues was significantly lower compared to that in normal tissues. However, miR-126 exhibited no difference between gastric cancer and normal tissues. A multivariate analysis demonstrated that the expression of miR-143 and -195 were associated with clinicopathological parameters, including depth of invasion and lymph node metastasis. This association may be applicable to future decisions regarding treatment or as a diagnostic biomarker.
\end{abstract}

\section{Introduction}

Gastric cancer is the fourth most common human malignant disease and the second most frequent cause of cancer-related

Correspondence to: Professor Chen Huang, Medical College, Xi'an Jiaotong University, 76 Yanta West Road, Xi'an, Shaanxi 710061, P.R. China

E-mail: hchen@mail.xjtu.edu.cn

Key words: miRNA, gastric cancer, clinical significance, biomarker mortality worldwide, causing $~ 800,000$ deaths annually. Approximately two-thirds of gastric cancer cases occur in developing countries, with $42 \%$ occurring in China alone (1). Although the optimal combination of surgical and non-surgical approaches has been used to treat gastric cancer, a considerable number of patients develop metastases to other sites due to the lack of reliable diagnostic techniques for early-stage detection (2). Therefore, diagnosis in the early stages is crucial for the selection of the most effective treatment for gastric cancer patients.

MicroRNAs (miRNAs) are small, non-coding RNAs, 19-24 nucleotides in length, which were first described by Lee et al (3). The mature single-stranded microRNAs bind to the $3^{\prime}$ untranslated region of potentially hundreds of target genes of imperfect complementarity, resulting in degradation of target mRNAs and inhibition of translation (4). Over the past 10 years, accumulating evidence strongly supports the role of miRNAs in crucial cellular processes, including development, differentiation, stress response, apoptosis and proliferation (5). Particularly in tumors, miRNAs may function as oncogenes and/or tumor suppressor genes (6).

Thus far, apart from traditional methods, the molecular technique is considered the optimal method for early diagnosis and prognosis prediction in cancer. Recent studies demonstrated that miRNAs are frequently dysregulated in human malignancies (7), including increased expression [i.e., miR-221 (8), -223 (9) and -135a (10)] and decreased expression [i.e., miRNA-200c (11), -451 (12) and -34b (13)]. This altered miRNA expression pattern provided novel opportunities for the use of biomarkers in cancer diagnosis (14). Several miRNA analyses were reported in human malignancies and the differences in expression between tumor tissues and their benign counterparts may be useful for cancer diagnosis (15). However, more ideal biomarkers or co-markers for gastric cancer are required for optimizing treatment selection for patients.

In this study, we compared the expression of 8 miRNAs (miR-21, -103, -106a, -126, -143, -195, -221 and -222) between gastric cancer and normal gastric tissue and investigated the 
association between their expression and clinical significance in order to identify potential diagnostic markers for gastric cancer.

\section{Materials and methods}

Sample collection. Gastrectomy samples were obtained from gastric cancer patients who underwent total gastrectomy at the Shaanxi Provincial People's Hospital and the First Affiliated Hospital of Xi'an Jiaotong University between 2009 and 2011. A total of 20 paired samples (gastric cancer and normal tissues, at a distance $>5 \mathrm{~cm}$ from the tumor) were randomly selected and independently examined. The pathological characteristics of the patients are summarized in Table I.

Isolation of total RNA from formaldenhyde-fixed, paraffin-embedded (FFPE) tissue. Total RNA was isolated from FFPE tissue sections with RecoverAll ${ }^{\mathrm{TM}}$ Total Nucleic Acid Isolation kit (Ambion, Austin, TX, USA) according to the manufacturer's instructions. Briefly, the sections were deparaffinized with xylene. Following addition of digestion buffer and protease, the samples were incubated at $50^{\circ} \mathrm{C}$ for $15 \mathrm{~min}$ and at $80^{\circ} \mathrm{C}$ for $15 \mathrm{~min}$. Subsequently, isolation additive and $100 \%$ ethanol were added. After washing 3 times, RNA was eluted in $60 \mu \mathrm{l}$ of elution solution and stored at $-80^{\circ} \mathrm{C}$.

Reverse transcription and quantitative polymerase chain reaction $(q P C R)$. RNA was reverse-transcribed to cDNA by priming with a mixture of looped primers and preamplified according to the manufacturer's instructions. The primers for miRNA were designed by our team (Table II) and commercially obtained from AuGCT Biotechnology Corporation (AuGCT, Beijing, China). The PrimeScript ${ }^{\circledR}$ RT reagent kit (Perfect Real Time; Takara Bio Inc., Shiga, Japan) was used. The reverse transcriptase reactions contained 20 ng of RNA samples, $2 \mu 1$ of $5 \mathrm{X}$ PrimeScript ${ }^{\circledR}$ Buffer (for Real Time), $0.5 \mu 1$ of PrimeScript ${ }^{\circledR}$ RT Enzyme Mix I, $4.5 \mu 1$ of RNase-free water and $1 \mu \mathrm{l}$ of stem-loop RT primers. The $10-\mu 1$ reactions were incubated for $15 \mathrm{~min}$ at $37^{\circ} \mathrm{C}$ and for $5 \mathrm{sec}$ at $85^{\circ} \mathrm{C}$ and maintained at $4^{\circ} \mathrm{C}$.

qPCR was performed using SYBR ${ }^{\circledR}$-Green I with the SYBR $^{\circledR}$ Premix Ex Taq ${ }^{\mathrm{TM}}$ II (Perfect Real Time). The qPCR reactions included $1 \mu \mathrm{l}$ of RT product dilution (100 ng), $10 \mu \mathrm{l}$ of $2 X$ SYBR Premix Ex Taq II, $7 \mu \mathrm{l}$ of RNase-free water, $1 \mu \mathrm{l}$ of forward primer and $1 \mu \mathrm{l}$ of reverse primer. U6 was used as an internal control to normalize the expression levels of the target genes. The qPCR was initiated with an initial denaturation step at $95^{\circ} \mathrm{C}$ for $30 \mathrm{sec}, 40$ cycles of $5 \mathrm{sec}$ at $95^{\circ} \mathrm{C}$ and $30 \mathrm{sec}$ at $60^{\circ} \mathrm{C}$. All the reactions were run in triplicate using the IQ-5 Real-Time PCR system (Bio-Rad Laboratories, Inc., Hercules, CA, USA).

The $\Delta \mathrm{Ct}$ and $2^{-\Delta \Delta \mathrm{Ct}}$ methods were used for analysis. The $\Delta \mathrm{Ct}$ value was calculated by the difference between the $\mathrm{Ct}$ values of the specific miRNA and U6: $\Delta \mathrm{Ct}=\mathrm{Ct}_{\text {miRNA }}-\mathrm{Ct}_{\mathrm{U} 6}$. $\Delta \Delta \mathrm{Ct}=\Delta \mathrm{Ct}_{\text {cancer tissues }}-\Delta \mathrm{Ct}_{\text {normal tissues }}$. The value of $2^{-\Delta \mathrm{Ct}}$ represented the miRNA expression of each sample and the value of $2^{-\Delta \Delta C t}$ represented the relative quotient (RQ) of the expression of the target gene to that of the control. In the present study, $\mathrm{RQ}$ represented the value of the ratio of miRNA expression in cancer tissue to that in normal tissue. $\mathrm{An} R \mathrm{Q}<1$ indicated
Table I. Summary of pathological characteristics of samples.

\begin{tabular}{|c|c|}
\hline Pathological characteristics & $\begin{array}{l}\text { Patient no. } \\
\quad(n=20)\end{array}$ \\
\hline \multicolumn{2}{|l|}{ Age (years) } \\
\hline$<60$ & 7 \\
\hline$>60$ & 13 \\
\hline \multicolumn{2}{|l|}{ Depth of invasion (T) } \\
\hline $\mathrm{T} 1$ & 2 \\
\hline $\mathrm{T} 2$ & 4 \\
\hline $\mathrm{T} 3$ & 3 \\
\hline $\mathrm{T} 4$ & 11 \\
\hline \multicolumn{2}{|l|}{ Lymph node metastasis $(\mathrm{N})$} \\
\hline Negative (N0) & 2 \\
\hline Positive (N1-N3) & 18 \\
\hline \multicolumn{2}{|c|}{ Haematogenous metastasis (M) } \\
\hline Negative (M0) & 17 \\
\hline Positive (M1) & 3 \\
\hline \multicolumn{2}{|l|}{ Stage } \\
\hline I & 2 \\
\hline II & 5 \\
\hline III & 7 \\
\hline IV & 6 \\
\hline \multicolumn{2}{|l|}{ Differentiation } \\
\hline Poor & 12 \\
\hline Moderate & 6 \\
\hline High & 2 \\
\hline
\end{tabular}

that the miRNA expression levels in cancer tissue were lower compared to those in normal tissue. Conversely, an RQ>1 indicated higher miRNA expression in cancer compared to normal tissues.

Statistical analysis. Data were analyzed with SPSS software, version 12.0 (SPSS Inc., Chicago, IL, USA) and Excel software (Microsoft, Redmond, USA). The paired samples t-test was used to compare the expression of miRNAs between cancer and normal tissues. One-way ANOVA was used to investigate the association between cancer and normal tissues. $\mathrm{P}<0.05$ was considered to indicate a statistically significant difference.

\section{Results}

Dysregulated miRNAs in cancer and normal tissues of gastric cancer patients. Among the 20 paired samples, 19 cases (95\%) exhibited a higher expression of miR-21, 15 cases (75\%) exhibited a higher expression of miR-103 and -106a and 13 cases (65\%) exhibited a higher expression of miR-221 and -222 in gastric cancer tissues compared to that in normal tissues (Fig. 1), whereas miR-126 exhibited no identical or significant differences between gastric cancer and normal tissues (higher in 10 and lower in the remaining 10 cases). Furthermore, miR-143 was shown to be decreased in 15 of the 20 pairs (75\%) (Fig. 2A). The average fold change of miR-143 between 
Table II. Primers for reverse transcription (RT) quantitative polymerase chain reaction of 8 microRNAs and U6.

\begin{tabular}{|c|c|}
\hline miRNAs & Primer sequences \\
\hline \multicolumn{2}{|l|}{ miR-103 } \\
\hline Forward: & ATCCAGTGCGTGTCGTG \\
\hline Reverse: & TGCTAGCAGCATTGTACAGG \\
\hline RT: & GTCGTATCCAGTGCGTGTCGTGGAGTCGGCAATTGCACTGGATACGACTCATAGC \\
\hline \multicolumn{2}{|l|}{ miR-106a } \\
\hline Forward: & ATCCAGTGCGTGTCGTG \\
\hline Reverse: & TGCTAAAAGTGCTTACAGTG \\
\hline RT: & GTCGTATCCAGTGCGTGTCGTGGAGTCGGCAATTGCACTGGATACGACCTACCTG \\
\hline \multicolumn{2}{|l|}{ miR-143 } \\
\hline Forward: & CAGTGCGTGTCGTGGAG \\
\hline Reverse: & GCGGTGAGATGAAGCACT \\
\hline RT: & GTCGTATCCAGTGCGTGTCGTGGAGTCGGCAATTGCACTGGATACGACGAGCTAC \\
\hline \multicolumn{2}{|l|}{ miR-145 } \\
\hline Forward: & CAGTGCGTGTCGTGGAGT \\
\hline Reverse: & AGGTCCAGTTTTCCCAGG \\
\hline RT: & GGAGTCGGCAATTGCACTGGATACGACAGGGATT \\
\hline \multicolumn{2}{|l|}{ miR-195 } \\
\hline Forward: & CAGTGCGTGTCGTGGAGT \\
\hline Reverse: & ACGGTAGCAGCACAGAAATA \\
\hline RT: & GGAGTCGGCAATTGCACTGGATACGACGCCAATA \\
\hline \multicolumn{2}{|l|}{ miR-21 } \\
\hline Forward: & ATCCAGTGCGTGTCGTG \\
\hline Reverse: & TGCTTAGCTTATCAGACTG \\
\hline RT: & TGGAGTCGGCAATTGCACTGGATACGACTCAACAT \\
\hline \multicolumn{2}{|l|}{ miR-221 } \\
\hline Forward: & ATCCAGTGCGTGTCGTG \\
\hline Reverse: & TGCTAGCTACATTGTCTGCT \\
\hline RT: & GTCGTATCCAGTGCGTGTCGTGGAGTCGGCAATTGCACTGGATACGACGAAACCC \\
\hline \multicolumn{2}{|l|}{ miR-222 } \\
\hline Forward: & ATCCAGTGCGTGTCGTG \\
\hline Reverse: & TGCTAGCTACATCTGGCT \\
\hline RT: & GTCGTATCCAGTGCGTGTCGTGGAGTCGGCAATTGCACTGGATACGACACCCAGT \\
\hline \multicolumn{2}{|l|}{ U6 } \\
\hline Forward: & GCTTCGGCAGCACATATACTAAAAT \\
\hline Reverse: & CGCTTCACGAATTTGCGTGTCAT \\
\hline RT: & CGCTTCACGAATTTGCGTGTCAT \\
\hline
\end{tabular}



Figure 1. Dysregulated expression of 8 miRNAs in gastric cancer compared to normal gastric tissues. Each dot represents a result of $2^{-\Delta \Delta C t}$ in one patient. Each experiment was performed in triplicate. gastric cancer and normal tissues was $1.121( \pm 1.589)$. miR-195 was also decreased in 16 of the 20 pairs (80\%) (Fig. 2B). The average fold change of miR-195 between gastric cancer and normal tissues was $0.799( \pm 0814)$.

Association between miRNA level and clinicopathological factors in patients with gastric cancer. To further investigate the roles of miR-143 and -195 expression in gastric cancer progression, the association between the expression of miR-143 (Fig. 3A) and -195 (Fig. 3B) was analyzed by one-way ANOVA. A decreased expression of miR-143 and -195 in gastric cancer was not associated with age $(>60$ vs. $<60$ years), lymph node metastasis (negative vs. positive), stage and differentiation (poor vs. moderate vs. high). However, miR-143 and -195 underexpression was found to be significantly associated with depth of invasion $(\mathrm{P}=0.000$ 
A

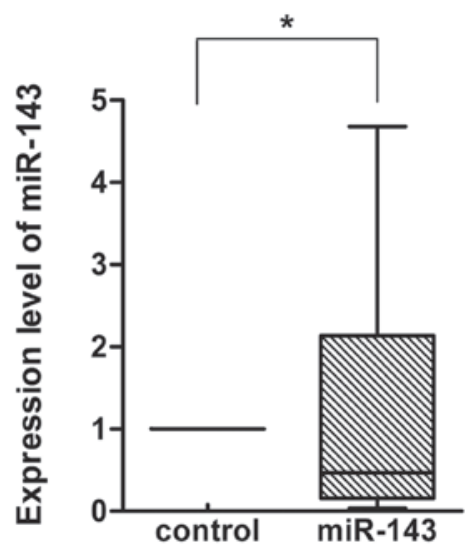

B

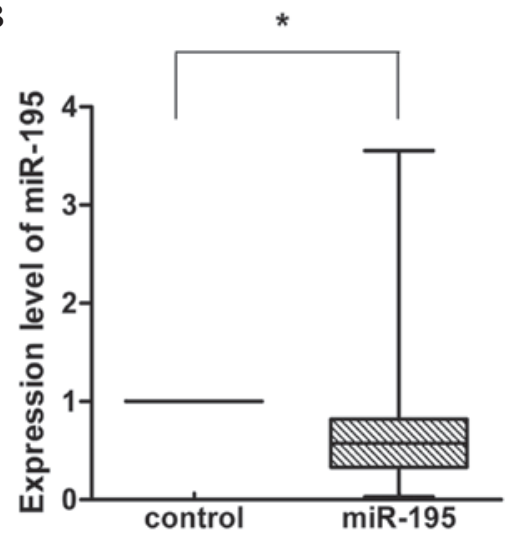

Figure 2. Underexpression of miR-143 and -195 in gastric cancer compared to normal tissues. (A) miR-143 was decreased in 15 of the 20 pairs (75\%) and (B) miR-195 was decreased in 16 of the 20 pairs $(80 \%)$. ${ }^{*} \mathrm{P}<0.05$. Each experiment was performed in triplicate.

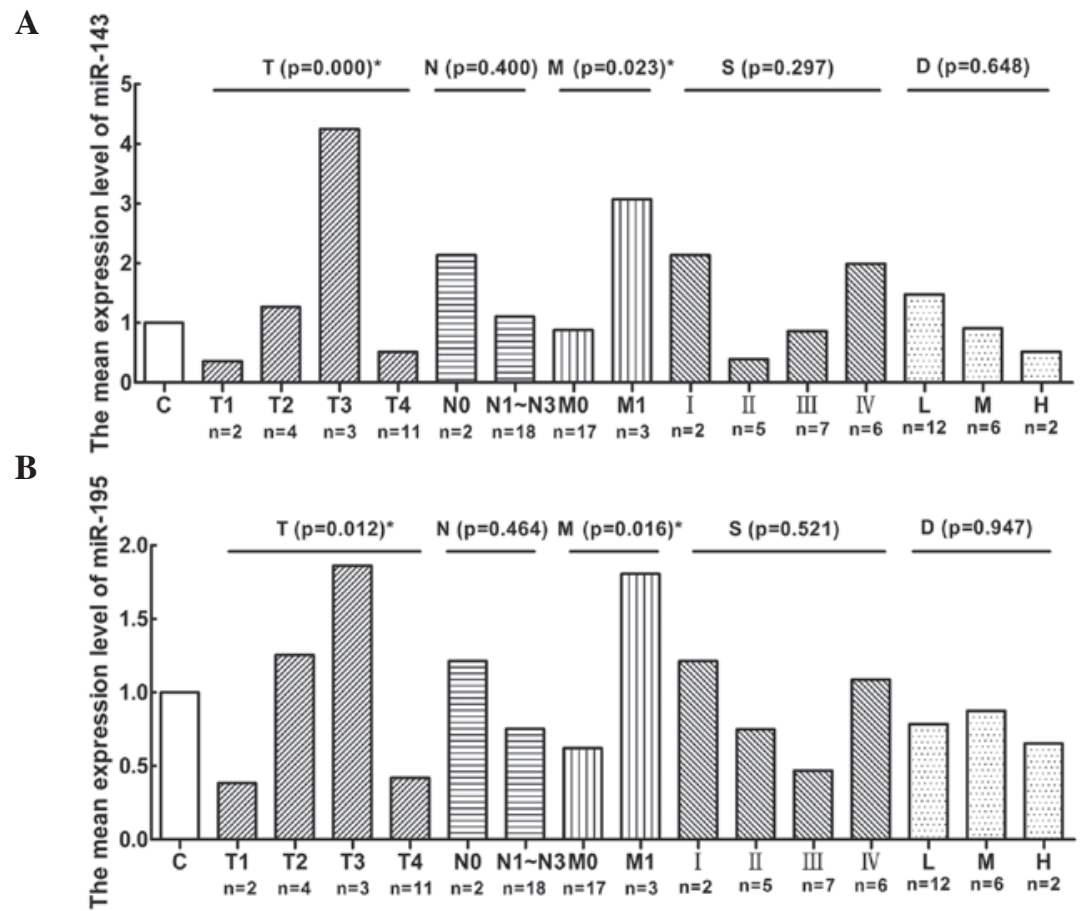

Figure 3. Association of expression levels of miR-143 and -195 with clinicopathological factors. (A) miR-143 underexpression as well as (B) miR-195 underexpression were significantly associated with depth of invasion and haematogenous metastasis. ${ }^{*} \mathrm{P}<0.05$.

and $\mathrm{P}=0.012$, respectively) and haematogenous metastasis $(\mathrm{P}=0.023$ and $\mathrm{P}=0.016$, respectively).

\section{Discussion}

Alterations in miRNA expression have been described in various cancer types and shown to be significantly correlated with cancer progression (16). miRNAs may function as oncogenes in human cancer. Our data demonstrated that miR-21, -103, -106a, -221 and -222 exhibited a higher expression in gastric cancer compared to normal gastric tissues (Fig. 1). The expression level of miR-21 was reported to be significantly higher in breast cancer compared to normal breast tissues in 109 patients who underwent surgery between 2002 and 2004; its overexpression was associated with mastectomy, larger tumor size, advanced stage, higher grade, estrogen receptor-negative status, human epidermal growth factor receptor 2-positive status, higher Ki-67 expression and mortality and it was significantly associated with lower overall survival (17). miR-103 expression was shown to be increased in patients with colorectal cancer (CRC) and was associated with poor prognosis; its actions are mediated through targeting the known metastasis suppressors death-associated protein kinase and Kruppel-like factor 4 in CRC cells, resulting in increased cell motility and cell-matrix adhesion and decreased cell-cell adhesion and epithelial marker expression (18). The expression analysis of miR-106a in the patient samples demonstrated its overexpression in CRC, leading to downregulation of the retinoblastoma protein activity, which plays an important role in cell cycle progression and, therefore, may be involved in malignant transformation of colonic cells (19). miR-221 and -222 were found to be overexpressed in several human 
cancers, including breast (20), prostate (21), lung (22) and liver cancer (23). Although the expression levels of miR-126 in CRC tissues were significantly lower compared to those in normal tissues and miR-126 overexpression may inhibit the growth of cancer cells (24), it was also found to be overexpressed in acute myeloid leukemia (25). Although the precise mechanisms underlying the biological functions of miRNAs have not been fully elucidated, our data demonstrated that miR-21, -103, $-106 \mathrm{a},-221$ and -222 were overexpressed in gastric cancer, which may indicate their role as oncogenes in cancerous processes.

Furthermore, previous studies reported the expression alterations and potential target sites of miR-143 and -195. Motoyama et al (26) used a miRNA microarray containing 455 human miRNA probes to determine the miRNA pattern in human $\mathrm{CRC}$ and the results demonstrated that the expression of miR-143 in cancer tissues was significantly lower compared to that in normal tissues. In addition, miRNA microarray analysis demonstrated that miR-143 was significantly downregulated, which may promote apoptosis and inhibit tumor formation by targeting Bcl-2 in cervical cancer (27). Ozata et al (28) reported that miR-195 was underexpressed in adrenocortical carcinoma (ACC) compared to normal adrenal cortices and benign adenomas. The overexpression of miR-195 may reduce cell proliferation and lead to significant induction of cell death in human NCI-H295R ACC cells. miR-195 was also found to be downregulated in CRC compared to normal colorectal tissue samples, with this downregulation being observed more frequently among patients with lymph node metastasis and advanced tumor stage (29). As regards the potential for a miRNA-based therapy, several target genes of miR-143 and -195 have already been proven experimentally, such as hexokinase 2 isoform and extracellular signal-regulated kinase-5 $(30,31)$ and WEE1 (32), respectively. Our data also demonstrated that miR-143 and -195 were downregulated in gastric cancer and were associated with depth of invasion (T) and haematogenous metastasis (M), providing a potential target for research of human cancer.

The potential usefulness of miRNA-based biomarkers for the diagnosis of numerous human cancers has been established by several studies. miR-222 expression in the urine was verified by in situ hybridization, which provided a high-accuracy method for bladder cancer diagnosis (33). As regards breast cancer, the differential expression of systemic miRNA-195 provides a possibility for detecting non-invasive and early-stage disease as a sensitive, specific, non-invasive cancer biomarker (34). However, the combination of markers is more reliable compared to a single marker for cancer diagnosis (35). The expression level of miR-126 in the patient serum was found to be associated with soluble mesothelin-related peptides, which are a specific marker of malignant pleural mesothelioma (MPM). miR-126 expression was correlated with a high risk of developing MPM and may be used as a marker for the early detection of MPM (36). This study identified miR-143 and -195 as potentially useful biomarkers in human gastric cancer, due to their similar performance in the clinicopathological factor analysis. We also suggest a potential diagnostic biomarker combination of miR-143 and -195 (co-markers) to improve their diagnostic sensitivity and specificity.
To validate the performance of biomarkers for the detection of cancer, further studies are required to verify whether the miRNAs we selected bear a full potential as either biomarkers or therapeutic targets in gastric cancer. However, we demonstrated that miR-143 and -195 , alone or in combination, may play a critical role in cancer progress and may constitute optimal biomarkers for the diagnosis of gastric cancer.

\section{Acknowledgements}

This study was supported by a grant from the Key Science and Technology Program of Shaanxi (no. 2010ZDKG-50), and the Program for Changjiang Scholars and Innovative Research Team in University (PCSIRT: 1171).

\section{References}

1. Parkin DM, Bray F, Ferlay J and Pisani P: Global cancer statistics, 2002. CA Cancer J Clin 55: 74-108, 2005.

2. Hohenberger P and Gretschel S: Gastric cancer. Lancet 362: 305-315, 2003.

3. Lee RC, Feinbaum RL and Ambros V: The C. elegans heterochronic gene lin-4 encodes small RNAs with antisense complementarity to lin-14. Cell 75: 843-854, 1993.

4. Wang XJ, Reyes JL, Chua NH and Gaasterland T: Prediction and identification of Arabidopsis thaliana microRNAs and their mRNA targets. Genome Biol 5: R65, 2004.

5. Karp X and Ambros V: Developmental biology. Encountering microRNAs in cell fate signaling. Science 310: 1288-1289, 2005.

6. Mraz M, Pospisilova S, Malinova K, Slapak I and Mayer J: MicroRNAs in chronic lymphocytic leukemia pathogenesis and disease subtypes. Leuk Lymphoma 50: 506-509, 2009.

7. Jiang Q, Wang Y, Hao Y, et al: miR2Disease: a manually curated database for microRNA deregulation in human disease. Nucleic Acids Res 37: D98-D104, 2009.

8. Dahiya N, Sherman-Baust CA, Wang TL, et al: MicroRNA expression and identification of putative miRNA targets in ovarian cancer. PLoS One 3: e2436, 2008.

9. Li X, Zhang Y, Zhang H, et al: miRNA-223 promotes gastric cancer invasion and metastasis by targeting tumor suppressor EPB41L3. Mol Cancer Res 9: 824-833, 2011.

10. Chen Y, Zhang J, Wang H, et al: miRNA-135a promotes breast cancer cell migration and invasion by targeting HOXA10. BMC Cancer 12: 111, 2012.

11. Shimono Y, Zabala M, Cho RW, et al: Downregulation of miRNA-200c links breast cancer stem cells with normal stem cells. Cell 138: 592-603, 2009.

12. Wang XC, Tian LL, Jiang XY, et al: The expression and function of miRNA-451 in non-small cell lung cancer. Cancer Lett 311: 203-209, 2011.

13. Lee YM, Lee JY, Ho CC, et al: miRNA-34b as a tumor suppressor in estrogen-dependent growth of breast cancer cells. Breast Cancer Res 13: R116, 2011.

14. Wang J, Chen J, Chang P, et al: MicroRNAs in plasma of pancreatic ductal adenocarcinoma patients as novel blood-based biomarkers of disease. Cancer Prev Res (Phila) 2: 807-813, 2009.

15. Paranjape T, Slack FJ and Weidhaas JB: MicroRNAs: tools for cancer diagnostics. Gut 58: 1546-1554, 2009.

16. He L, Thomson JM, Hemann MT, et al: A microRNA polycistron as a potential human oncogene. Nature 435: 828-833, 2005.

17. Lee JA, Lee HY, Lee ES, Kim I and Bae JW: Prognostic implications of microRNA-21 overexpression in invasive ductal carcinomas of the breast. J Breast Cancer 14: 269-275, 2011.

18. Chen HY, Lin YM, Chung HC, et al: miR-103/107 promote metastasis of colorectal cancer by targeting the metastasis suppressors DAPK and KLF4. Cancer Res 72: 3631-3641, 2012.

19. Catela Ivkovic T, Aralica G, Cacev T, Loncar B and Kapitanovic S: miR-106a overexpression and $\mathrm{pRB}$ downregulation in sporadic colorectal cancer. Exp Mol Pathol 94: 148-154, 2013.

20. Stinson S, Lackner MR, Adai AT, et al: TRPS1 targeting by miR-221/222 promotes the epithelial-to-mesenchymal transition in breast cancer. Sci Signal 4: ra41, 2011. 
21. Sun T, Yang M, Chen S, et al: The altered expression of MiR-221/-222 and MiR-23b/-27b is associated with the development of human castration resistant prostate cancer. Prostate 72: 1093-1103, 2012

22. Zhang Y, Ma T, Yang S, et al: High-mobility group A1 proteins enhance the expression of the oncogenic miR-222 in lung cancer cells. Mol Cell Biochem 357: 363-371, 2011.

23. Yoo BK, Santhekadur PK, Gredler R, et al: Increased RNA-induced silencing complex (RISC) activity contributes to hepatocellular carcinoma. Hepatology 53: 1538-1548, 2011.

24. Li XM, Wang AM, Zhang J and Yi H: Down-regulation of miR-126 expression in colorectal cancer and its clinical significance. Med Oncol 28: 1054-1057, 2011.

25. Li Z and Chen J: In vitro functional study of miR-126 in leukemia. Methods Mol Biol 676: 185-195, 2011.

26. Motoyama K, Inoue $\mathrm{H}$, Takatsuno $\mathrm{Y}$, et al: Over- and under-expressed microRNAs in human colorectal cancer. Int J Oncol 34: 1069-1075, 2009

27. Liu L, Yu X, Guo X, et al: miR-143 is downregulated in cervical cancer and promotes apoptosis and inhibits tumor formation by targeting Bcl-2. Mol Med Report 5: 753-760, 2012.

28. Ozata DM, Caramuta S, Velazquez-Fernandez D, et al: The role of microRNA deregulation in the pathogenesis of adrenocortical carcinoma. Endocr Relat Cancer 18: 643-655, 2011.

29. Wang X, Wang J, Ma H, Zhang J and Zhou X: Downregulation of miR-195 correlates with lymph node metastasis and poor prognosis in colorectal cancer. Med Oncol 29: 919-927, 2012.
30. Peschiaroli A, Giacobbe A, Formosa A, et al: miR-143 regulates hexokinase 2 expression in cancer cells. Oncogene 32: 797-802, 2013.

31. Clape C, Fritz V, Henriquet C, et al: miR-143 interferes with ERK5 signaling, and abrogates prostate cancer progression in mice. PLoS One 4: e7542, 2009.

32. Bhattacharya A, Schmitz U, Wolkenhauer O, Schonherr M, Raatz Y and Kunz M: Regulation of cell cycle checkpoint kinase WEE1 by miR-195 in malignant melanoma. Oncogene 32: 3175-3183, 2012.

33. Puerta-Gil P, Garcia-Baquero R, Jia AY, et al: miR-143, miR-222, and miR-452 are useful as tumor stratification and noninvasive diagnostic biomarkers for bladder cancer. Am J Pathol 180: $1808-1815,2012$

34. Heneghan HM, Miller N, Kelly R, Newell J and Kerin MJ: Systemic miRNA-195 differentiates breast cancer from other malignancies and is a potential biomarker for detecting noninvasive and early stage disease. Oncologist 15: 673-682, 2010

35. Liang QL, Shi HZ, Qin XJ, Liang XD, Jiang J and Yang HB: Diagnostic accuracy of tumour markers for malignant pleural effusion: a meta-analysis. Thorax 63: 35-41, 2008.

36. Santarelli L, Strafella E, Staffolani S, et al: Association of MiR-126 with soluble mesothelin-related peptides, a marker for malignant mesothelioma. PLoS One 6: e18232, 2011. 